Tropical Journal of Pharmaceutical Research March 2019; 18 (3): 625-630

ISSN: $1596-5996$ (print); 1596-9827 (electronic)

(C) Pharmacotherapy Group, Faculty of Pharmacy, University of Benin, Benin City, 300001 Nigeria.

\title{
Effect of rosuvastatin and benazepril on matrix metalloproteinase-2, matrix metalloproteinase-9 and leukotriene B4 of patients with acute myocardial infarction
}

\author{
Li Sai ${ }^{1}$, Zhang YanQiu ${ }^{2}$, Cui DongMei ${ }^{2}$, Li YinJun ${ }^{1 *}$ \\ ${ }^{1}$ Department of Cardiovascular Medicine, The 4th People's Hospital of Shenyang, Shengyang City, ${ }^{2}$ Department of \\ Cardiovascular Medicine, Huludao Central Hospital, Huludao City, China
}

*For correspondence: Email: vu1286@163.com

\begin{abstract}
Purpose: To investigate the effects of rosuvastatin and benazepril on matrix metalloproteinase-2 (MMP-2), MMP-9 and leukotriene B4 (LTB4) of patients with acute myocardial infarction (AMI).

Methods: Fifty-six patients with AMI were selected. They were randomly divided into control and study groups. Thirty healthy people were used in the normal group. On the basis of conventional therapy, patients in the control group were given rosuvastatin orally, while those in the study group received rosuvastatin and benazepril orally. The duration of treatment in both groups was 3 months. Serum levels of MMP-2, MMP-9 and LTB4, and incidence of left ventricular remodelling and recurrence of cardiovascular events were determined before and after treatment for both groups.

Results: MMP-2, MMP-9 and LTB4 levels in serum were significantly lower for the two groups after treatment, when compared to pre-treatment values, and significantly lower in the study group $(p<0.05)$. Left ventricular remodelling was lower in the study group than in the control group $(p<0.05)$. Recurrence of cardiovascular events declined significantly in the study group, relative to control $(p>$ 0.05).

Conclusion: Rosuvastatin and benazepril significantly reduce serum levels of MMP-2, MMP-9 and LTB4 in AMI patients, and thus can potentially prevent ventricular remodelling, improve prognosis and reduce recurrence rate.
\end{abstract}

Keywords: Rosuvastatin, Benazepril, Acute myocardial infarction, MMP-2, MMP-9, LTB4

\begin{abstract}
This is an Open Access article that uses a funding model which does not charge readers or their institutions for access and distributed under the terms of the Creative Commons Attribution License (http://creativecommons.org/licenses/by/4.0) and the Budapest Open Access Initiative (http://www.budapestopenaccessinitiative.org/read), which permit unrestricted use, distribution, and reproduction in any medium, provided the original work is properly credited.
\end{abstract}

Tropical Journal of Pharmaceutical Research is indexed by Science Citation Index (SciSearch), Scopus, International Pharmaceutical Abstract, Chemical Abstracts, Embase, Index Copernicus, EBSCO, African Index Medicus, JournalSeek, Journal Citation Reports/Science Edition, Directory of Open Access Journals (DOAJ), African Journal Online, Bioline International, Open-J-Gate and Pharmacy Abstracts

\section{INTRODUCTION}

Acute myocardial infarction (AMI) is based on the formation of coronary atherosclerotic plaques. Fatigue, emotional and other factors cause intravascular plaque rupture and platelet aggregation around the plaque, resulting in coronary artery ischaemia and hypoxia that lead to myocardial necrosis [1]. The incidence, mortality and recurrence of $\mathrm{AMI}$ are relatively high, with approximately 500,000 AMI patients in China each year [2]. With rapid developments in modern society, AMI is rising year by year [3].

Atherosclerosis (AS) is the main pathological basis of $\mathrm{AMI}$, and the main factors that affect 
plaque formation and rupture are inflammation and abnormal lipid metabolism. Leukotriene B4 (LTB4) is involved in the formation of atherosclerotic plaques, and matrix metalloproteinases (MMPs) are associated with plaque instability. Leukotriene B4 (LTB4) also affects the expressions of MMPs in arterial plaques, resulting in the instability of atherosclerotic plaque [4]. Rosuvastatin is a new class of lipid-regulating drugs. Relative to other statin drugs, rosuvastatin has the advantages of high safety, few side effects and flexible administration time.

In this study, rosuvastatin and benazepril were used to treat patients with AMI. The serum levels of MMP-2, MMP-9 and LTB4, and incidence of left ventricular remodelling and recurrence of cardiovascular events were determined pre- and post-treatment.

\section{EXPERIMENTAL}

\section{Patients}

Fifty-six patients who were diagnosed with AMI in Huludao Central Hospital from May 2015 to October 2017 were selected. The time from onset to admission was $5.6 \pm 0.1 \mathrm{~h}$. Using a random number table, the patients were divided into a study group and a control group (28 patients each). The study group comprised 15 men and 13 women, with mean age of $53 \pm 1.2$ years, and mean onset time of $5.7 \pm 0.1 \mathrm{~h}$. In There were 14 men and 14 women in the control group (mean age of $54 \pm 0.8$ years), with onset time of $5.5 \pm 0.5 \mathrm{~h}$. The two groups were comparable in gender, age and disease duration between the two groups $(p>0.05)$.

Thirty healthy volunteers were used as normal control after subjecting them to physical examination in the hospital. They consisted of 18 men and 12 women of mean age of $51 \pm 1.2$ years. The diagnostic criteria used were Guide to Diagnosis and Treatment of Acute Myocardial Infarction developed by the Cardiology Branch of the Chinese Medical Association in 2001 [5]. The patients selected met at least two of the following three criteria: (1) clinical symptoms of acute ischaemic chest pain; (2) dynamic changes in serum myocardial markers associated with myocardial necrosis, and (3) dynamic changes in electrocardiogram (ECG). Patients who met the AMI diagnostic criteria i.e. onset time $<12 \mathrm{~h}, 18$ - 75 years old, male or female, and signed informed consent, were included.

Patients in the following categories were excluded (1) those with previous history of $\mathrm{MI}$; (2) patients who took statins and/or angiotensin converting enzyme inhibitors (ACEIs) within 2 weeks before admission; (3) patients with heart failure, autoimmune disease or hepato-renal insufficiency; (4) patients with cerebrovascular accidents or other neurological diseases; (5) patients who were allergic to statins, and (6) those undergoing percutaneous coronary intervention and/or intravenous thrombolytic therapy. This study received approval from the Ethical Committee of The 4th People's Hospital of Shenyang (Approval No. 4phs-201503221), and was executed in line with the Helsinki declaration of 1964 which was subjected to amendment in 1996 [6].

\section{Treatment methods}

\section{Conventional treatment}

The conventional AMI treatment for patients admitted to the hospital was sustained. The patients were placed on absolute bed rest, and were not permitted to take other ACEI drugs.

\section{Study group}

Patients in the study group were given $20 \mathrm{mg}$ rosuvastatin orally once per night within $24 \mathrm{~h}$ of admission, and $2.5 \mathrm{mg}$ benazepril orally once daily in the morning or evening. The control group received $20 \mathrm{mg}$ rosuvastatin within $24 \mathrm{~h}$ of admission once per night. The duration of treatment in both groups was 3 months.

\section{Assessment of serum markers}

Both groups received medication within $24 \mathrm{~h}$ after admission. Fasting cubital venous blood (6 $\mathrm{mL}$ ) was collected pre- and post-treatment. The samples were subjected to centrifugation at 1500 rpm for $10 \mathrm{~min}$, and assays for MMP-2, MMP-9 and LTB4 were carried out in the serum with an automated enzyme-linked immunoassay system (Tianjin Boko Bio-Industry Co., Ltd).

\section{Echocardiography indices}

All patients underwent echocardiography on day 1 and 3 months after admission. The left ventricular end-diastolic volume index (LVEDVI) and left ventricular end-diastolic volume index increase $(\triangle \mathrm{LVEDVI})$ were measured and corrected as in Eq 1.

$\Delta L=\{(C L-A L) / A L\} \ldots \ldots \ldots \ldots(1)$

where $\triangle L V E D V I$ is $\triangle L, C L$ is convalescent LVEDVI, and $A L$ is acute LVEDVI . 
Values of $\triangle$ LVEDVI $\leq 20 \%$ indicate that there was no left ventricular remodelling, while $\triangle$ LVEDVI $>20 \%$ indicate that there was left ventricular remodelling.

\section{Follow-up of patients}

After the treatment, regular follow-up was carried out once a month for a total of 6 months. The renal function, liver function and other indicators of regular outpatient monitoring were followed up. Rosuvastatin was stopped if the related indicators continued to increase abnormally $(>3$ times normal). The recurrence of cardiovascular events in all patients was recorded. All patients used a non-invasive cuff to monitor blood pressure.

\section{Statistical analysis}

Measured data are expressed as mean \pm standard deviation ( $\bar{x} \pm S D$ ). Counting data were analysed using the $\chi^{2}$ test. A normality test was performed first, and paired sample $t$-test was applied pre- and post-treatment in the groups. The comparison between the groups was carried out with independent sample $t$-test. All statistical evaluations were done with statistical software SPSS v19.0. Values of $p<0.05$ were taken as indicative of statistical significance.

\section{RESULTS}

\section{Serum levels of MMP-2, MMP-9 and LTB4}

Table 1 shows that serum MMP-2, MMP-9 and LTB4 were significantly higher in AMI patients than in control $(p<0.01)$.

Table 1: Serum levels of MMP-2, MMP-9 and LTB4 prior to treatment

\begin{tabular}{lccc}
\hline Group & $\begin{array}{c}\text { MMP-2 } \\
(\mathbf{p g} / \mathbf{L})\end{array}$ & $\begin{array}{c}\text { MMP-9 } \\
(\mathbf{p g} / \mathbf{L})\end{array}$ & $\begin{array}{c}\text { LTB4 } \\
(\mathbf{p g} / \mathbf{L})\end{array}$ \\
\hline Study & $812.10 \pm$ & $798.03 \pm$ & $614.06 \pm$ \\
& $152.01^{\mathrm{a}}$ & $172.03^{\mathrm{a}}$ & $66.35^{\mathrm{a}}$ \\
Control & $822.12 \pm$ & $804.09^{ \pm}$ & $624.36 \pm$ \\
& $148.11^{\mathrm{a}}$ & $152.04^{\mathrm{a}}$ & $59.15^{\mathrm{a}}$ \\
Normal & $212.11^{ \pm}$ & $210.09^{ \pm} \pm$ & $300.06 \pm$ \\
& $62.12^{2}$ & $72.07^{2}$ & 26.15 \\
\hline${ }^{a} P<0.01$, compared with patients in normal control \\
group
\end{tabular}

\section{Serum levels of MMP-2, MMP-9 and LTB4 post-treatment}

Table 2 shows that the serum levels of MMP-2, MMP-9 and LTB4 were reduced in both groups post-treatment $(p<0.05)$. However, MMP-2 and MMP-9 concentrations were lower in the serum of the study group, relative to control values $(p<$ $0.05)$.

Table 2: Serum levels of MMP-2, MMP-9 and LTB4 after treatment

\begin{tabular}{lcccc}
\hline Group & $\begin{array}{c}\text { Time- } \\
\text { point }\end{array}$ & $\begin{array}{c}\text { MMP-2 } \\
(\mathbf{p g} / \mathbf{L})\end{array}$ & $\begin{array}{c}\text { MMP-9 } \\
(\mathbf{p g} / \mathbf{L})\end{array}$ & $\begin{array}{c}\text { LTB4 } \\
\mathbf{( p g / L )}\end{array}$ \\
\hline & Before & $812.10 \pm$ & $798.03 \pm$ & $614.06 \pm$ \\
Study & & $152.01^{\mathrm{a}}$ & $172.03^{\mathrm{a}}$ & $66.35^{\mathrm{a}}$ \\
& After & $301.12 \pm$ & $298.11 \pm$ & $204.01 \pm$ \\
& & $106.11^{\mathrm{b}}$ & $110.05^{\mathrm{b}}$ & 33.12 \\
& Before & $822.12 \pm$ & $804.09 \pm$ & $624.36 \pm$ \\
Control & & $148.11^{\mathrm{a}}$ & $152.04^{\mathrm{a}}$ & $59.15^{\mathrm{a}}$ \\
& After & $399.28 \pm$ & $419.12 \pm$ & $217.18 \pm$ \\
& & 123.11 & 131.21 & 43.71
\end{tabular}

${ }^{a} P<0.05$, comparison before and after treatment in both groups; ${ }^{b} p<0.05$, study and control group compared after treatment

\section{Incidence of left ventricular remodelling after treatment}

After treatment, the incidence of left ventricular remodelling was reduced significantly in the study group $(p<0.05)$.

Table 3: Cases of left ventricular remodelling after treatment $(n=28)$

\begin{tabular}{lcc}
\hline Group & $\begin{array}{c}\text { Left ventricular } \\
\text { remodelling/case } \\
(\%)\end{array}$ & $\begin{array}{c}\text { Non-left ventricular } \\
\text { remodelling/case } \\
(\%)\end{array}$ \\
\hline Study & $6(21.4)^{a}$ & $22(78.6)^{a}$ \\
Control & $15(53.5)$ & $13(46.5)$ \\
\hline${ }^{a} P<0.05$, comparison of the incidence of left \\
ventricular remodelling between the groups
\end{tabular}

\section{Adverse events during follow-up}

After treatment, regular follow-up was carried out for 6 months. One patient in the study group had angina and another one had MI. In the control group, three patients presented with angina and three patients that had recurrent $\mathrm{MI}$ in the control group. Relative to controls, there were lower recurrence of cardiovascular events in the study group $(p>0.05)$.

Table 4: Follow-up $(n=28)$

\begin{tabular}{lcc}
\hline Group & $\begin{array}{c}\text { Number of } \\
\text { recurrences of } \\
\text { cardiovascular } \\
\text { events }(\mathbf{n})\end{array}$ & $\begin{array}{c}\text { Recurrence of } \\
\text { cardiovascular } \\
\text { events (\%) }\end{array}$ \\
\hline Study & 2 & $7.1^{\mathrm{a}}$ \\
Control & 6 & 21.4 \\
\hline${ }^{\mathrm{a}} P<0.05$ (comparison between the groups)
\end{tabular}

\section{Safety}

There were three patients with mild liver enzyme abnormalities in the study group during 
treatment, but there was no further elevation after resumption of medication. Two patients had gastrointestinal discomfort (mild nausea and anorexia), and two patients had mild liver enzyme abnormalities in the control group during treatment. However, there was no further elevation after resumption of medication.

\section{DISCUSSION}

Previous studies have shown that AS is closely related to inflammatory reactions. Due to stimulation of inflammatory factors, unstable atheromatous plaques in the intima of coronary arteries undergo fibrous cap degeneration. Longterm inflammatory reactions lead to fibrin cap rupture and ulceration, which in turn cause platelet aggregation, adhesion and thrombosis in the plaque rupture in the blood vessel. This results in coronary artery embolism and insufficient blood supply, leading to the development of $\mathrm{AMI}$, and it affects the clinical outcome and prognosis of AMI patients.

Inflammatory factors and inflammatory cells in atherosclerotic plaques are the key factors that cause plaque instability and rupture. They also cause some irreversible effects such as degradation of the extracellular matrix, expansion of the plaque lipid centre and the destruction of fibrous tissue [7].

MMPs are proteolytic enzymes that rely on zinc and calcium metal ions. They degrade the extracellular matrix within the plaque. Indeed, MMP-2 and MMP-9 are the most potent degrading factors of the extracellular matrix, and MMP-2 and MMP-9 are the key factors responsible for the instability of atherosclerotic plaques [8]. Type IV collagen is one of the substrates for MMP-2 and MMP-9. It is a component of the atheromatous basement membrane and the fibrous cap of unstable plaques. Type IV collagen influences the development of atheromatous plaques due to its degradation. However, MMP-2 and MMP-9 can degrade type IV collagen quickly.

With the onset of AMI, the expression of MMP-9 increases under hypoxic conditions, leading to accelerated degradation of collagen and myocardial fibrosis, compensatory ventricular dilation and ventricular remodelling [9]. Leukotriene B4 is an active product of arachidonic acid metabolism, and it has the highest content of LTs and the strongest effect amongst the 20-carbon unsaturated acids. The inflammatory response involved in LTB4 permits the occurrence and development of coronary AS. Related studies have shown that LTB4 increases the expressions of MMPs in plaques, making the degradation of fibrous caps faster than their formation, accelerating the degradation of the extracellular matrix and plaque rupture, and ultimately precipitating cardiovascular events [10].

Rosuvastatin is a selective, competitive HMGCoA reductase inhibitor. It promotes the decomposition and metabolism of low density lipoprotein (LDL) through inhibition of the synthesis of cholesterol precursors, and reduces the synthesis of cholesterol. Rosuvastatin has potent lipid-lowering, anti-inflammatory, plaquestabilising, vascular endothelial functionimproving and immune regulatory effects. It can prevent and treat multiple myocardial and cerebrovascular diseases such as MI and stroke. Research has shown that rosuvastatin can effectively reduce the incidence of left ventricular remodelling after $\mathrm{MI}$, and it could also reduce the risk of $\mathrm{Ml}$, delay the progression of heart failure and reduce cardiovascular mortality [11].

It has been reported that rosuvastatin significantly reduced serum levels of MMP-2 and MMP-9 in patients with AMI, relative to simvastatin [12]. The effects of rosuvastatin were superior to those of simvastatin, and its side effects were also fewer. In the current study, after 3 months of treatment with rosuvastatin in both groups, serum levels of MMP-2 and MMP-9 were significantly lower than their corresponding values pre-treatment.

Angiotensin converting enzyme inhibitors (ACEls) block angiotensin II receptors, reduce angiotensin II levels and inhibit the production of oxygen free radicals by inhibiting angiotensin converting enzyme (ACE). These effects reduce the expressions of MMPs and reduce inflammation, stabilise plaques, increase myocardial blood flow, protect against myocardial ischaemia and reduce the incidence of cardiovascular events. Angiotensin converting enzyme inhibitors can improve bradykinin levels, increase nitric oxide (NO) synthesis, improve vascular endothelial function, inhibit ventricular remodelling, exert long-term myocardial protection and improve outcomes and prognosis in patients with cardiovascular disease. Studies have shown that heart failure caused by coronary heart disease in patients with exhausted myocardial cells was associated with increased expression of MMP-9, and that benazepril significantly inhibited MMP-9 expression [13].

It has been reported that ACEls directly inhibit the serum activities of MMP-9 and MMP-2 $[14,15]$. The results obtained in this study 
suggest that benazepril downregulates the activity of MMP-2 and MMP-9. After treatment, the incidence of left ventricular remodelling was significantly lower in the study group than in the control group. It has been demonstrated that statin therapy can restore coronary function by increasing the level of endogenous $\mathrm{NO}$ and by significantly improving left ventricular remodelling after AMI [16].

The recurrence of cardiovascular events in the observation group was $7.1 \%$, in contrast to 21.4 $\%$ in the control group. The results showed that rosuvastatin and benazepril effectively reduced blood contents of MMP-2, MMP-9, LTB4 and other lipid mediators. These effects reduced the degradation of matrix components, stabilised plaques, reduced left ventricular remodelling, delayed the development of heart failure and reduced the recurrence of cardiovascular events. In the current treatment of acute coronary artery disease, the severity of coronary stenosis is promptly detected using coronary angiography. Coronary artery bypass grafting, coronary thrombectomy and other revascularisation techniques are used to correct severe coronary stenosis and improve acute coronary symptoms quickly and effectively. However, these methods cannot change the physio-pathological problems of the blood vessels. Thus, the essential problem of plaques in blood vessels persist. Therefore, it is important to determine the effective method for stabilising intravascular plaques in the treatment of AMI by investigating the pathological mechanisms underlying atherosclerotic plaque formation.

\section{Limitations of the study}

Few participants were included in this study. Moreover, a blank control group was not used in this research. Thus, there is need to apply the results and conclusion with caution.

\section{CONCLUSION}

The results obtained in this study have shown that rosuvastatin and benazepril significantly reduce blood concentrations of MMP-2, MMP-9 and LTB4 in AMI sufferers. Therefore, they can potentially prevent ventricular remodelling, improve prognosis and reduce recurrence in patients with acute myocardial infarction.

\section{DECLARATIONS}

\section{Conflict of Interest}

No conflict of interest associated with this work.

\section{Contribution of Authors}

We declare that this work was done by the authors named in this article and all liabilities pertaining to claims relating to the content of this article will be borne by the authors. The study was conceived and designed by $\mathrm{Li}$ YinJun; Zhang YanQiu. Cui DongMei collected and analysed the data, while $\mathrm{Li}$ Sai wrote the manuscript. All authors read and approved the text prior to publication.

\section{REFERENCES}

1. $\mathrm{Xu} K Y$. Research progress of integrated traditional Chinese and Western medicine in treating acute myocardial infarction. Chin J Clin Ration Drug Use 2017; 10(1): 178-179.

2. Gu SF, Sun N, Lian W. Research status of early rehabilitation treatment of acute myocardial infarction in the emergency percutaneous coronary intervention. Chin J Pract Nurs 2016; 32(11): 872-875.

3. Cheng $R$, Su $X Y$, He $Y Y$. Clinical significance of combined detection of three indexes in cardiocerebrovascular diseases. Lab Med Clin 2017; 14(3): 1074-1076.

4. Ma HG, Zhu FF, Wang YX. Study on the relationship between carotid atherosclerosis and serum LTB4, MMP9 levels in patients with acute cerebral infarction. China Med Herald 2017; 14: 52-55.

5. Yuan JQ, Song Y. Guidelines for diagnosis and treatment of acute ST segment elevation myocardial infarction in China in 2015: updated highlights. Chin Circ J 2016; 31(4): 318-320.

6. World Health Organization. Declaration of Helsinki. $\mathrm{Br}$ Med J 1996; 313(7070): 1448-1449.

7. Maracle CX, Agca R, Helder B, Meeuwsen JAL, Niessen HWM, Biessen EAL, de Winther MPJ, de Jager SCA, Nurmohamed MT, Tas SW. Noncanonical NF-kB signaling in microvessels of atherosclerotic lesions is associated with inflammation, atheromatous plaque morphology and myocardial infarction. Atherosclerosis 2018; 270: 33-41.

8. Opstad TB, Seljeflot I, Bøhmer E, Arnesen H, Halvorsen S. MMP-9 and Its Regulators TIMP-1 and EMMPRIN in Patients with Acute ST-Elevation Myocardial Infarction: A NORDISTEMI Substudy. Cardiology 2018; 139: 1724.

9. Wu ZY, Yao LP. Changes of serum MMP-9, TIMP-1 and TNF- $\alpha$ levels of patients with Kawasaki disease and its relationship with coronary artery lesions. J Community Med 2016; 14(1): 17-20.

10. Wang ZJ, Liang M, Zhu MH, Wen HY, Zhai JM, Yang XB. Determination and significance of leukotriene C4 and D4 in children with adenoidal hypertrophy. Guangdong Med J 2016; 37(5): 692-694.

11. Zhang LF, Jia XW, Geng HP, Feng HP. Effects of different doses of rosuvastatin on myocardial

Trop J Pharm Res, March 2019; 18(3):629 
reperfusion and cardiac function in patients undergoing percutaneous coronary intervention in acute myocardial infarction. Chin J Clin Pharmacol 2015; 4(13): 12231226.

12. Zuo BG, Song CL, Dang YB, Yan YJ. Effect of rosuvastatin on MMPs and inflammatory factors in patients with acute myocardial infarction. Chin Trop Med 2016; 10: 1032-1035.

13. Yokota $T$, Tomita $H$, Mori $Y$, Kudo $T$, Hiraga $H$, Suto $N$, Higuma $T$, Abe $N$, Hanada $H$, Osanai $T$, et al. Imidapril and enalapril similarly inhibit plasma matrix metalloproteinase activities and attenuate left ventricular remodeling in patients with acute myocardial infarction. J Cardiovasc Pharmacol 2014; 63(6): 528-532.

14. Ramírez R, Díez J, Sanmartín M, Saura M, Zamorano JL, Zaragoza C. Nanotechnology Applied to Preserve
Extracellular Matrix as Teranostic Tool in Acute Myocardial Infarction. Rev Esp Cardiol 2018; 9: 18855857.

15. Kong $P$, Shinde AV, Su Y, Russo I, Chen B, Saxena A, Conway SJ, Graff JM, Frangogiannis NG. Opposing Actions of Fibroblast and Cardiomyocyte Smad3 Signaling in the Infarcted Myocardium. Circ 2018; 137(7): 707-724.

16. Kim JS, Kim W, Park JY, Woo JS, Lee TW, Ihm CG, Kim YG, Moon JY, Lee SH, Jeong MH, et al. Effects of statin therapy on clinical outcomes after acute myocardial infarction in patients with advanced renal dysfunction: $A$ propensity score-matched analysis. Plos One 2017; 12(8): 0183059. 